\title{
Effect of Follicle Size on Metaphase II and Cleavage Rate of Goat Oocyte
}

\author{
$\underline{\text { Sri Wahjuningsih }}{ }^{1, a)}$ and Muhammad Nur Ihsan ${ }^{1}$ \\ 1)Laboratory of Animal Reproduction, Faculty of Animal Husbandry, University of \\ Brawijaya,Indonesia

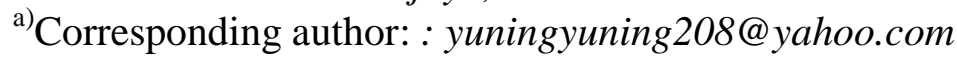

\begin{abstract}
The aim of this research was to determine the effect of follicle size on Metaphase II and cleavage rate of goat Oocyte. Ovaries from slaughterhouse transported to the laboratory within $2 \mathrm{~h}$ of slaugtering in normal saline. The ovaries were cut and classified two classes of follicle size : small (2-4 mm diameter ) and large (>4-8 $\mathrm{mm}$ diameter) follicles. All culture were done in ten replicates at $38.5^{\circ} \mathrm{C}, 5 \% \mathrm{CO}_{2}, 95 \%$ humidity. Only fully Cumulus Oocyte Complexes (COCs) and homogenous cytoplasm were used. The COCs were matured for $26 \mathrm{~h}$ in TCM-199 medium supplemented with FCS $10 \%+$ EBS $10 \%$. Chi square test were used for statistical analysis. Metaphase II rate was determined by microscope in a sample of COCs denuded, fixed and stained with acetoorcein $1 \%$. In vitro fertilization was performed using frozen semen $\left(2 \times 10^{6}\right.$ spermatozoa $/ \mathrm{mL}$ in EBSS solution. The percentage of COCs reaching Metaphase II was $86.42 \%(261 / 302)$ and $74.33 \%$ (220/300) for oocytes originating from large and small diameter follicles,respectively $(\mathrm{P}<0.05)$. After in vitro fertilization, the developmental competence of oocytes dependently increased with follicular size. Higher cleavage rate was obtained from large follicles compare to small follicles $(\mathrm{P}<0.05)$.
\end{abstract}

Keywords : Follicle size,Metaphase II, Goat oocyte

\section{INTRODUCTION}

In vitro embryo production of embryos represents the third generation of techniques aimed at a better control of animal reproduction. This techniques involved major steps : oocyte collection, oocyte in vitro maturation, in vitro fertilization and in vitro development of the resulting embryos.It is well recognized that oocytes recovered from slaughterhouse materials for in vitro embryo production are extremely heterogenous in terms of quality and developmental competence. Diversity of follicular development may affect the oocyte quality and that oocyte would be at different developmental stages when derived from different sized follicle. The developmental competence of follicular oocytes in vitro may be affected by the follicular sizes (Majeed et al., 2012; El Shaahat et al., 2013). The characteristics of follicles could be an important parameter to determine the developmental competence of oocytes fertilized in vitro. The aim of this research was to determine the effect of follicle size on metaphase II and cleavage rate of goat oocyte.

\section{METHODS}

Ovaries from slaughterhouse transported to the laboratory within $2 \mathrm{~h}$ of slaugtering in normal saline. The ovaries were cut and classified two classes of follicle size : small (2-4 $\mathrm{mm}$ diameter $)$ and large ( $>4-8 \mathrm{~mm}$ diameter) follicles. All culture were done in ten 
replicates at $38.5^{\circ} \mathrm{C}, 5 \% \mathrm{CO}_{2}, 95 \%$ humidity. Only fully Cumulus Oocyte Complexes (COCs) and homogenous cytoplasm were used. The COCs were matured for $26 \mathrm{~h}$ in TCM-199 medium supplemented with FCS $10 \%$ + EBS 10\%. Chi square test were used for statistical analysis. Metaphase II rate was determined by microscope in a sample of COCs denuded,fixed and stained with acetoorcein $1 \%$. In vitro fertilization was performed using frozen semen $\left(2 \times 10^{6}\right.$ spermatozoa/mL in EBSS solution).

\section{RESULT}

The percentage rate of metaphase II oocyte and cleavage rate were greater for oocyte from large follicle compare to those from small follicle $(\mathrm{P}<0.05)$

Table 1. The percentage of metaphase-II oocyte and cleavage rate in different follicular size

\begin{tabular}{lll} 
Follicular size & \multicolumn{1}{c}{ Metaphase-II oocyte $(\%)$} & Cleavage rate $(\%)$ \\
\hline $2-<4 \mathrm{~mm}$ & $220 / 300(74.33)^{\mathrm{a}}$ & $110 / 202(54.46)$ \\
$4-6 \mathrm{~mm}$ & $261 / 302(86.42)^{\mathrm{b}}$ & $183 / 255(71.76)$ \\
\hline
\end{tabular}

Value in the same column with different letters differ significantly $(\mathrm{p}<0.05)$

\section{DISCUSSION}

Oocyte maturation was a process of cytoplasmic maturation that comes with maturation of the cell nucleus. Oocyte maturation process can be carried out based on the level of meiosis, the process of cell division of gametes from diploid to haploid nature. Oocyte meiotic process can be observed through the nucleus in the form of phase transformation: Germinal vesicle $(\mathrm{GV})$, germinal vesicle Break Down (GVBD), Metaphase I to Metaphase II.The maturation rate of oocytes collected from small and large follicles based on metaphase II rate was 74.33 $\%$ and $86.42 \%$,respectively. Our results showed higher maturation rate in oocytes from large follicles as compared with the small follicle oocytes. The higher number of metaphase II oocyte recovered from large follicles compared to small follicles might be attributed to the development of oocyte in large follicles.

Meiotic competence is acquired progressively during follicular growth. Full maturation involves both nuclear and cytoplasmic events that confer on the oocyte capacity for supporting normal fertilization and early embryonic development. Oocytes accumulate a very stable form of RNA that is translated during maturation, fertilization and early embryonic development (Khatir et al.,2007). The RNA accumulation could be well influenced by the quality of follicular microenvironment. Wani et al., (2013) reported that a factor inherent in oocytes derived from smaller follicles limited their further development. Follicle size can greatly affect oocyte developmental competence and concluded that the factors required for improved development are found within cytoplasm of the oocytes derived from the larger follicles. The transcript produced during this period of low transcriptional activity are critical for development and the improved of developmental competence is due to cytoplasmic maturation of the oocyte during the final phase of folliculogenesis. Such follicle although not preovulatory are presumably among those competing for dominance and thus may provide an environment more conducive to proper cytoplasmic maturation, giving their oocytes greater capacity for embryonic development.

It was reported that a factor inherent in oocytes derived from smaller follicles limited their further development in bovine. Follicle size can greatly affect oocyte developmental competence and concluded that the factors required for improved development are found 
within cytoplasm of the oocytes derived from the larger follicles (Topleret al., 2016). It was reported that even though only cumulus oocyte complexes (COCs) with uniform ooplasm and compact cumulus cell mass were usually selected for oocyte maturation, there was a large variation in the dictyate stage of the first meiotic prophase among oocyte collected from follicles in different sizes. Therefore, the quality of oocytes collected from different follicular size or different ages might be a major of variation in IVM results.

The competence to undergo cytoplasmic maturation is also acquired progressively by oocyte during follicular growth. Therefore possible explanations for relationship between follicular size and oocyte quality may be : 1) a factor inherent in the oocytes derived from the smaller follicles limits their further development, 2)the cytoplasm of oocyte derived from the larger follicles may have some factors required for improved development and 3) the larger follicle may provide a better environment to induce cytoplasmic maturation for supporting subsequent embryonic development.

In vitro embryo development is known to be highly affected by oocytes quality. In present research, the cleavage rates observed were $54.46 \%$ and $71.76 \%$ from small and large follicle oocytes, respectively which were significantly different $(\mathrm{P}<0.05)$. The results support the hypothesis that there was higher developmental competence of oocytes from large follicles than small follicles in goat.

\section{CONCLUSIONS}

The percentage of COCs reaching Metaphase II and cleavage rate of oocytes increased with greater follicular size diameter. Higher Metaphase II and cleavage rate were obtained from large follicles compare to small follicles $(\mathrm{P}<0.05)$

\section{REFERENCES:}

El Shaahat, ElMaaty, A.M., Moawad A.R., 2013. Follicular Fluid Composition in Relation to Follicular Size in Pregnant and Non Pregnat Dromedary Camels (Camelus dromedaries). Anim. Reprod 10(1) : 16-23

Khatir,H., Anouassi A., Tibary, A., 2007. Effect of Follicular Size on in Vitro developmental Competence of Oocytes and Viability of Embryos After Transfer in The Dromedary (camelus dromedaries. Animal Reproduction Science 99 : 413-420

Majeed,A.F.,AlSaigh M.N., Al- Timimi,I.H.S., 2012. Effect of Follicular Size on Recovery and In Vitro Maturation of Black Iraqi Goat Oocytes. Al-Anbar J Vet. Sci 5 (1) : 130-133

Topler,D.,Ebeling S., Weitzel J.M., Spannbrucker A.C., 2016. Effect of Follicle Size on In Vitro Maturation of Pre-Pubertal Porcine Cumulus Oocyte Complexes. Reproduction in Domestic Animals 51(3) : 370-377

Wani,A.R., Khan,M.Z., Sofi, K.A. Malik, A.a, Lone FA and Bhat,F.A., 2013. Effect of Follicular Size on In Vitro Maturation and Culture of Sheep Embryos. Iranian J.of Vet.Rechearch 14 (4) : 299-304 